\title{
Level density parameters
}

\section{T. von Egidy*}

Physik-Department, Technische Universität München, D-85748 Garching, Germany

E-mail: egidy@ph.tum.de

\section{H.-F. Wirth}

Physik-Department, Technische Universität München, D-85748 Garching, Germany

E-mail: wirth@tum.de

\section{Bucurescu}

National Institute of Physics and Nuclear Engineering, R-76900 Bucharest, Romania

E-mail: bucurescultandem.nipne.ro

Parameters for nuclear level density formulas were determined for 310 nuclei from $\mathrm{F}$ to $\mathrm{Cf}$ applying the Back-Shifted Fermi Gas model (BSFG) and the Constant Temperature model (CT). The experimental parameters can be reproduced by simple formulas containing only values from atomic mass tables. These formulas can be used to predict unknown level densities.

Workshop on Photon Strength Functions and Related Topics

June 17-20 2007

Prague, Czech Republic

${ }^{*}$ Speaker. 


\section{Introduction}

Nuclear level densities contain the essential information on nuclei at higher excitation energies, especially when individual levels cannot be resolved anymore. In addition to this fundamental property, they are needed for the calculation of reaction rates, for the determination of photon strength functions, in astrophysics, and for fission and fusion reactor design. Although the simple relation between mass and energy is well known, it is not obvious that the level density at higher excitation energies depends mainly on the ground state masses as will be shown in this contribution. Details of our studies can be found in Ref. [1].

\section{Level density formulas}

The exponential increase of the level density can be described by the Back-Shifted Fermi Gas formula (BSFG) with the two free parameters $a$ and $E_{1}$ or by the Constant Temperature formula (CT) with the parameters $T$ and $E_{0}$. These two free parameters can be determined for each nucleus by a fit to the known individual levels in a given energy and spin range at low excitation energies and by the neutron resonance density at the neutron binding energy. Additionally a formula for the spin distribution has to be assumed. We have done this for 310 nuclei between ${ }^{19} \mathrm{~F}$ and ${ }^{251} \mathrm{Cf}$. For most of them the neutron resonance density was known from IAEA reports [2]. The 310 low energy level schemes were taken from the Nuclear Data Sheets, from the Table of Isotopes [3], and from special publications and were individually checked for the limits of completeness. The following level density formulas were used:

$$
\rho(E, J, \pi)=\frac{1}{2} \rho(E) f(J),
$$

where $E$ is the excitation energy, $J$ the spin, and $\pi$ the parity. The parity dependence was neglected. The spin dependence was assumed to be

$$
f(J)=\exp \left(-J^{2} / 2 \sigma^{2}\right)-\exp \left[-(J+1)^{2} / 2 \sigma^{2}\right]
$$

with $\sigma$ being the spin cut-off parameter.

The formula for the BSFG model is

$$
\begin{aligned}
\rho_{\mathrm{BSFG}}(E) & =\exp \left[2 \sqrt{a\left(E-E_{1}\right)}\right] /\left[12 \sqrt{2} \sigma a^{1 / 4}\left(E-E_{1}\right)^{5 / 4}\right], \quad \text { with } \\
\sigma^{2} & =0.0146 A^{5 / 3}\left[1+\sqrt{1+4 a\left(E-E_{1}\right)}\right] / 2 a .
\end{aligned}
$$

The CT model uses the formulas

$$
\rho_{C T}(E)=\exp \left[\left(E-E_{0}\right) / T\right] / T \quad \text { and } \quad \sigma=0.98 A^{0.29} .
$$

Figure 1 shows the experimental and fitted curves of the cumulative numbers of levels for eight nuclei as a function of the excitation energy. It should be emphasized that the resonance density is also included in the fit. The agreement is very good. Only the first five levels are not so well reproduced. But our fit procedure [1] gives a low weight to the first levels. All obtained parameters, $a$ and $E_{1}$ for BSFG, and $T$ and $E_{0}$ for CT are listed in Ref. [1] and shown in Figs. 2, 3, and 4. 


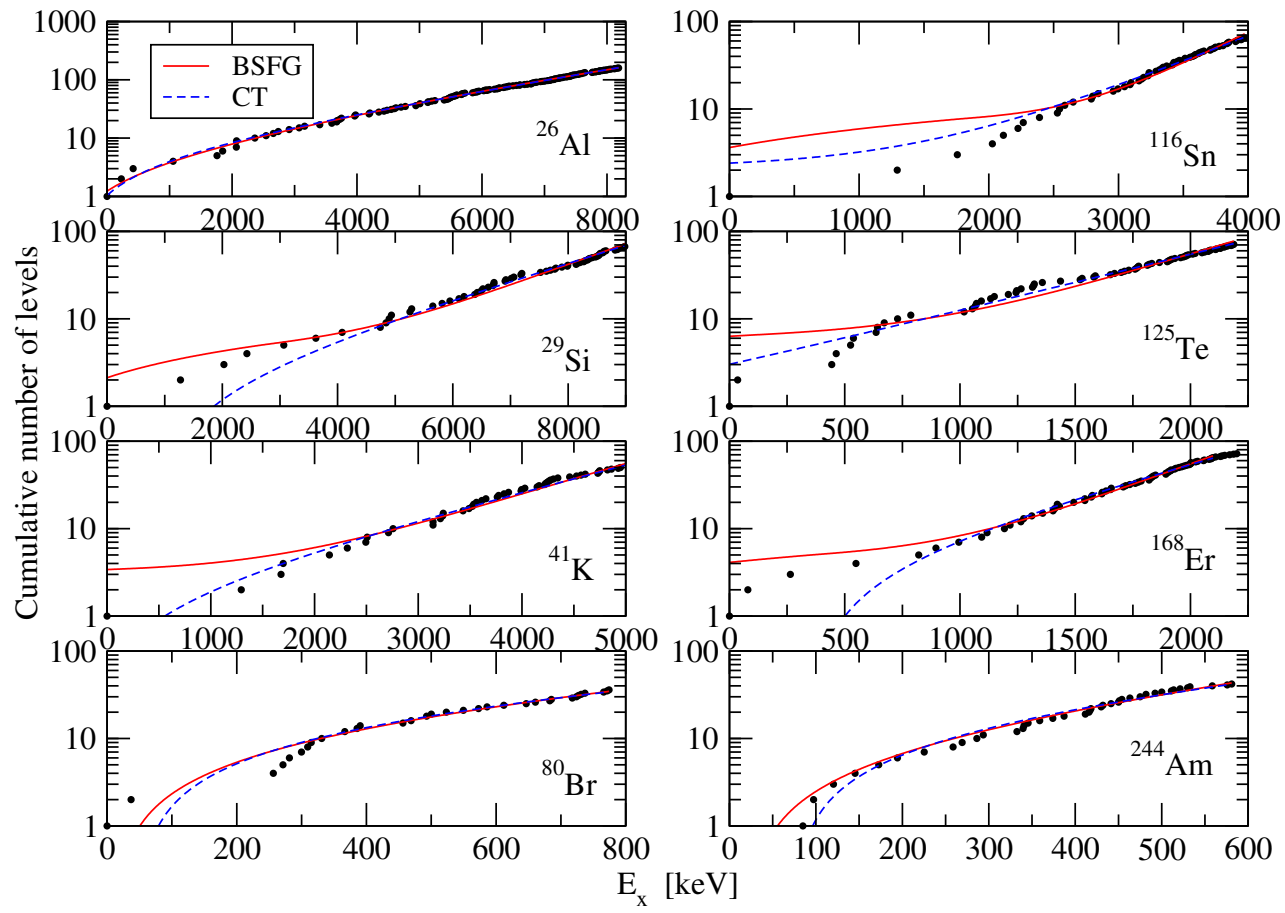

Figure 1: Experimental and fitted cumulative numbers of levels as a function of the excitation energy for eight nuclei. The fits were made with the BSFG and CT models and include the level spacing at the neutron binding energy.

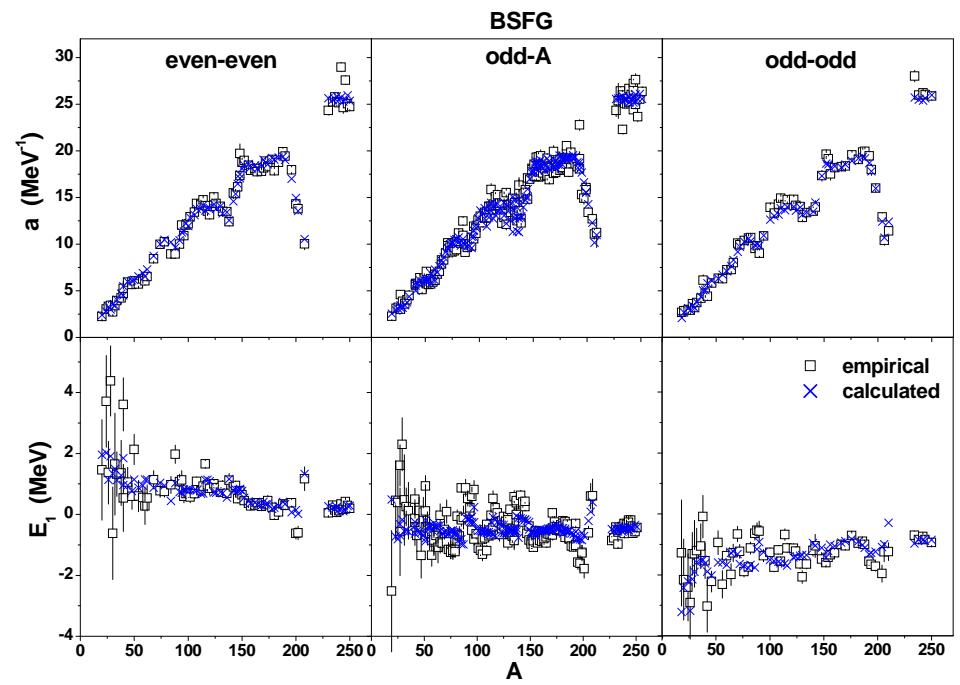

Figure 2: Experimental and fitted level density parameters for the BSFG model as a function of the mass number $A$. 


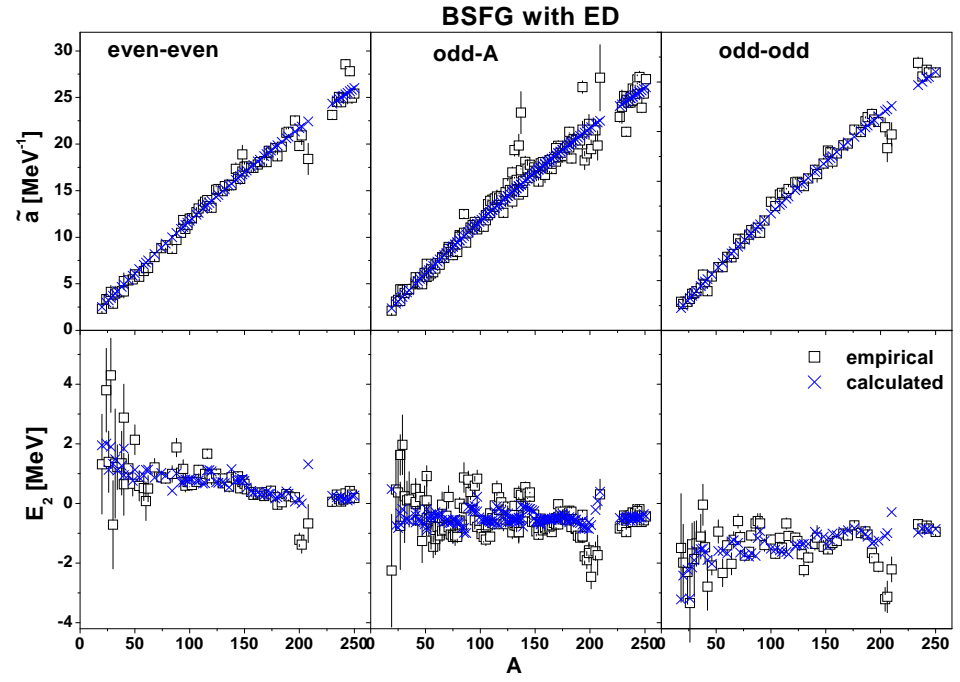

Figure 3: The same as Fig. 2 but for the BSFG model with energy dependent $a$.

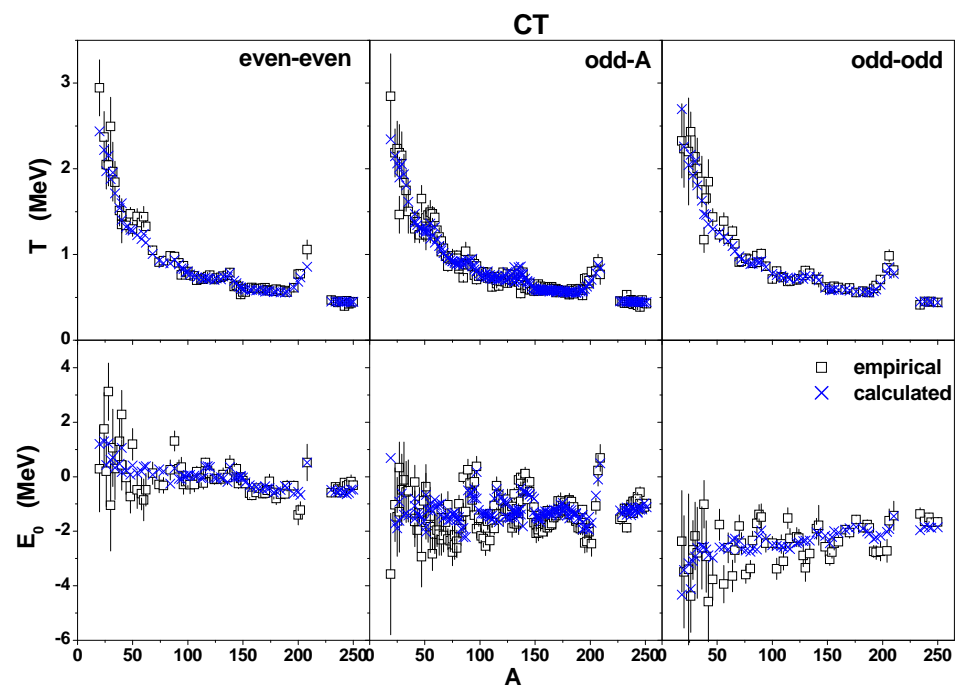

Figure 4: The same as Fig. 2 but for the CT model.

\section{Formulas for level density parameters}

In the next step we made a careful search for correlations of the level density parameters with well known observables such as mass number $A$, ground-state mass, pairing energy, and shell effect. We found that the pairing correction is best reproduced by the deuteron pairing energy:

$$
P a=\frac{1}{2}(-1)^{Z}[-M(A+2, Z+1)+2 M(A, Z)-M(A-2, Z-1),
$$

where $M$ is the experimental mass or mass excess value. For the shell correction $S$ we used the mass $M_{\mathrm{ld}}$ of the liquid-drop mass formula of J.M. Pearson [4]:

$S=M-M_{\mathrm{ld}} \quad$ and $\quad S^{\prime}=S-\Delta, \quad$ with

$\Delta=+0.5 P a, 0,-0.5 P a \quad$ for even-even, odd, and odd-odd nuclei, respectively. 

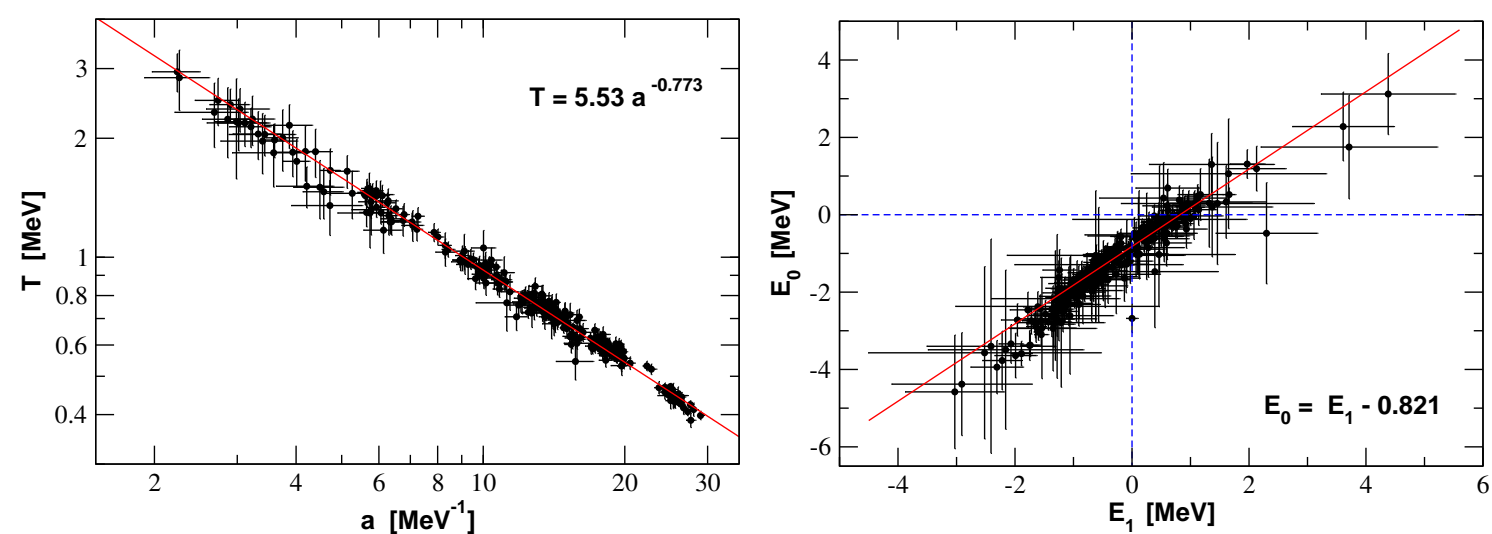

Figure 5: Correlations between the level density parameters $a$ and $T$, and between $E_{1}$ and $E_{0}$.

Our result is that the level density parameters of the 310 nuclei can be well reproduced using very simple formulas containing from atomic mass tables only $\mathrm{A}, \mathrm{Pa}, \mathrm{S}^{\prime}$, and

$$
d S / d A=[S(Z+1, N+1)-S(Z-1, N-1)] / 4 .
$$

The formulas are for the BSFG model:

$$
\begin{aligned}
& a=A^{0.90}\left(0.1848+0.00828 S^{\prime}\right) \quad \text { and } \\
& E_{1}=-0.48-0.5 P a+0.29 d S / d A \text { for even-even, } \\
& E_{1}=-0.57-0.5 P a+0.70 d S / d A \text { for even-odd, } \\
& E_{1}=-0.57+0.5 P a-0.70 d S / d A \text { for odd-even, } \\
& E_{1}=-0.24+0.5 P a+0.29 d S / d A \text { for odd-odd, }
\end{aligned}
$$

and for the CT model

$$
\begin{aligned}
T=A^{-2 / 3} /\left(0.0571+0.00193 S^{\prime}\right) & \text { and } \\
E_{0}=-1.24-0.5 P a+0.33 d S / d A & \text { for even-even, } \\
E_{0}=-1.33-0.5 P a+0.90 d S / d A & \text { for even-odd, } \\
E_{0}=-1.33+0.5 P a-0.90 d S / d A & \text { for odd-even, } \\
E_{0}=-1.22+0.5 P a+0.33 d S / d A & \text { for odd-odd. }
\end{aligned}
$$

A.V. Ignatyuk et al. [5] suggested that the shell dependence of the parameter $a$ of the BSFG model should decrease with increasing excitation energy. We applied also their formulas:

$$
\begin{aligned}
& a(E, Z, N)=\tilde{a}\left[1+S^{\prime}(Z, N) f\left(E-E_{2}\right) /\left(E-E_{2}\right)\right] \quad \text { and } \\
& f\left(E-E_{2}\right)=1-\exp \left[-\gamma\left(E-E_{2}\right)\right], \quad \text { with } \gamma=0.06 \mathrm{MeV}^{-1} .
\end{aligned}
$$

These formulas reduce the shell effect very slowly, $10 \%$ at $10 \mathrm{MeV}, 50 \%$ at $30 \mathrm{MeV}$.

Using Ignatyuk's formulas the experimental level densities can be fitted with the extremely simple formulas:

$$
\tilde{a}=0.1847 A^{0.90} \quad \text { and } \quad E_{2}=E_{1} .
$$


Figures 2, 3, and 4 demonstrate that these formulas are able to reproduce most of the level density parameters. Consequently, the new simple formulas can be applied to calculate level density parameters of nuclei where experimental values are not available.

\section{Correlations of level density parameters}

Furthermore, we investigated the correlations between the level density parameters of the BSFG and CT formulas and found the simple relations:

$$
T=5.53 a^{-0.773} \quad \text { and } \quad E_{0}=E_{1}-821 \mathrm{keV}
$$

Figure 5 demonstrates these relations. Since the experimental results show that $T \sim A^{-2 / 3}$ and the above relation gives $a \sim T^{-1.294}$, we can calculate $a \sim A^{(-2 / 3)(-1.294)}=A^{0.863}$. This is close to the value $A^{0.90}$ in our new formula.

\section{Comparison with previous level density parameters}

Two frequently quoted publications, by A.S. Iljinov et al. [6] and by T. Rauscher et al. [7], present calculations of level density parameters. There are several important differences to our calculations:

1. Data set: Iljinov et al. use low level groups, resonance densities, and reaction data.

Rauscher et al. take only resonance densities.

We include complete low energy level schemes and resonance densities.

2. Backshift: Iljinov et al. use $\Delta=\chi 12 / \sqrt{A}$ with $\chi=0,1,2$ for odd-odd, odd, and even-even nuclei, respectively.

Rauscher et al. apply $\Delta=\frac{1}{2}\left(\Delta_{\mathrm{n}}+\Delta_{\mathrm{p}}\right)$, with pairing gaps from mass tables.

We fit the backshift as free parameter and relate these values then to the deuteron pairing energies.

3. Formulas: The different authors use slightly different formulas for the spin cut-off parameter and for the shell correction. All authors use Ignatyuk's formulas. Iljinov et al. test also collective effects (rotation, vibration).

We apply also the BSFG formula with constant $a$ and the CT formula.

4. Fit procedures: Iljinov et al. calculate the $a$ parameter for each experimental level density and then fit the parameters of $\tilde{a}$ to all $a$ values.

Rauscher et al. determine the three free parameters of $\tilde{a}$ by a fit to all resonance densities.

We obtain in a first fit the level density parameters $a, E_{1}$ and $\tilde{a}, E_{2}$ and $T, E_{0}$ for each nucleus and in a second step we try to reproduce these parameters as functions of the pairing and shell energies from mass tables. 


\section{Conclusion}

A new set of experimental level density parameters was determined for 310 nuclei from ${ }^{19} \mathrm{~F}$ to ${ }^{251} \mathrm{Cf}$. Very simple formulas are proposed to calculate these parameters from mass table values. This means that the level density at higher excitation energies depends mainly on ground state mass values. The formulas can be used to predict level densities where experimental data does not exist. The formulas have been tested up to about $10 \mathrm{MeV}$. They have to be compared with experimental results at higher energies to find out, up to which energies they can be applied. It is a challenge for the theory to confirm the found relations.

\section{References}

[1] T. von Egidy and D. Bucurescu, Phys. Rev. C 72, 044311 (2005); Phys. Rev. C 72, 067304 (2005); Phys. Rev. C 73, 049901 (E) (2006).

[2] G. Reffo, Rep. IAEA-TECDOC-1034, Vienna 1998, p. 25 and RIPL-2 database: http://www. nds.iaea.org.

[3] R.B. Firestone and V.S. Shirley, Table of Isotopes, John Wiley and Sons, Inc., New York 1996.

[4] J.M. Pearson, Hyperfine Interactions 132, 59 (2001).

[5] A.V. Ignatyuk, G.N. Smirenkin, and A.S. Tishin, Sov. J. Nucl. Phys. 21, 255 (1975); A.V. Ignatyuk, K.K. Istekov, and G.N. Smirenkin, Sov. J. Nucl. Phys. 29, 450 (1979).

[6] A.S. Iljinov, V.M. Mebel, N. Bianchi, E. De Sanctis, C. Guaraldo, V. Lucherini, V. Muccifora, E. Polli, A.R. Reolon, and P. Rossi, Nucl. Phys. A543, 517 (1992).

[7] T. Rauscher, F.-K. Thielemann, and K.-L. Kratz, Phys. Rev. C 56, 1613 (1997). 